\title{
Modify the rheological properties of Daura asphalt by thermoplastic polyurethane using the microwave technique
}

\author{
Salam J. Marzina ${ }^{1 *}$, Khalid A. Owaid ${ }^{2}$ \\ ${ }^{1,2}$ Department of Chemistry, College of Education for Pure Sciences, University of Mosul, Mosul, Iraq \\ E-mail: ${ }^{1 *}$ salamkarash@gmail.com, Khalid.a.waid73@gmail.com ${ }^{2}$
}

(Received February 09, 2020; Accepted May 01, 2020; Available online September 01, 2020)

DOI: 10.33899/edusj.2020.126651.1047, @ 2020, College of Education for Pure Science, University of Mosul.

This is an open access article under the CC BY 4.0 license (http://creativecommons.org/licenses/by/4.0/).

\begin{abstract}
:
There is a growing need to produce asphalt materials with high economic feasibility, that is the production of asphalt materials with rheological properties that differ from the base asphalt (unmodified asphalt), can be used in many fields such as the production of paving asphalt, where it is not appropriate to use ordinary asphalt.

This study included the modification of the rheological properties of Daura asphalt by catalytic chemical treatment with thermoplastic polyurethane (Used in the interior parts of cars) using a microwave oven at a power of (360) watts and at different periods of time. As well as treat asphalt samples with the thermoplastic polyurethane in the presence of sulfur.

The modified asphalt samples were studied in terms of ductility, penetration, softening point and penetration Index.

A sample with good rheological specification was selected $\left(\mathrm{As}_{7}\right)$ that are conform with the specifications of Iraqi Roads and Bridges Authority, The selected sample were studied in terms of conducting Marshall tests and comparing them with the original sample in order to demonstrate the possibility of using the modified sample as an paving asphalt. The obtained results showed good specifications in terms of crawl and stability values.

An aging test was also conducted on the sample $\left(\mathrm{As}_{7}\right)$ of the good rheological specifications.
\end{abstract}

Key world: modified-asphalt; thermoplastic polyurethane; rheological properties.

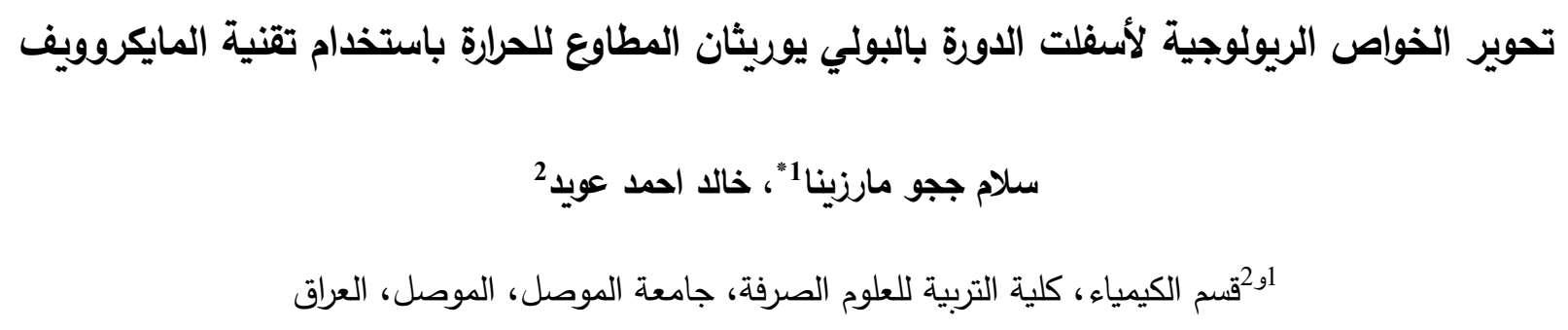




\section{Journal of Education and Science (ISSN 1812-125X), Vol: 29, No: 3, 2020 (85-101)}

الخلاصة

نظراً للحاجة الماسة لإنتاج مواد إسفلتية ذات جدوى اقتصادية عالية تتمثل في إنتاج مواد اسفلتية تصلح للاستخدام في مجالات كثيرة كإنتاج إسفلت تبليط ذو صفات ريولوجية جيدة حيث من غير المناسب استخدام الاسفلت الأساس غير المحور . تضمنت هذه الدراسة تحوير الخواص الريولوجية لأسفلت الدورة بالمعالجة الكيميائية المحفزة مع البولي يوريثان المطاوع للحرارة (المستخدم في الاجزاء الداخلية للسيارات) باستخدام فرن المايكروويف بطاقة (360) واط وبفترات زمنية مختلفة ، كما تمت معالجة هذه الخواص مع البولي يوريثان المطاوع للحرارة بوجود الكبريت. ومن ثم تمت دراسة عينات الاسفلت المحورة (الخواص الريولوجية) من خلال إجراء فحوصات النفاذية ، والاستطالة ، ودرجة الليونة ودليل الاختراق. تم اختيار عينة ذات المواصفات الريولوجية جيدة (As $)$ والتي تتوافق مع مواصفات هيئة الطرق والجسور العراقية، ودراسة العينة

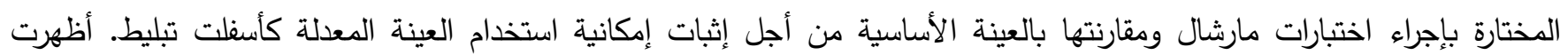
النتائج التي تم الحصول عليها مواصفات جيدة من حيث قيم الزحف والاستقرارية، كما وأجري ايضاً فحص التقادم على النموذج (As ذي المواصفات الريولوجية الجيدة.

الكلمات المفتاحية: الإسفلت المحور، البولي يوريثان المطاوع للحرارة، الصفات الريولوجية.

المقدمة

يعرف الأسفلت بأنه مادة سائلة ثقيلة ذات لزوجة تزداد بانخفاض درجة الحرارة حتى تصل حدود الصلابة ويتكون بدرجة رئيسة من هيدروكاربونات ذوات تراكيب بارافينية ونفينية وارومية، كما يشتمل على مركبات حلقية أو غير حلقية تحتوي على النتروجين،

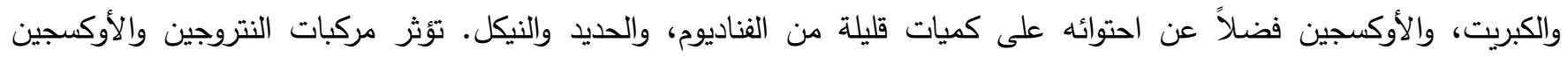
والكبريت مع العناصر المعدنية في الصفات الفيزيائية للاسفلت، إذ تعمل العناصر المعدنية القطبية على زيادة التداخل بين الجزئيات

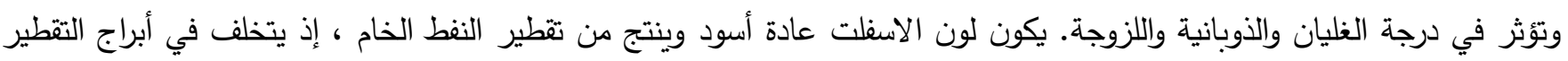
بهيئة مادة ثقيلة بعد أن يتم سحب المواد الخفيفة والمتطايرة [1, 2]. إن طبيعة الصفات الفيزيائية للاسفلت تحدد طبيعة الاستعمال أكثر من تلك الكيميائية ، وذلك بسبب استخدامه والمواد المصنَعَة منه في التطبيقات والأعمال الهندسية [3,4]. وهناك عدة مواصفات مُعتمَدَة للبتيومين قد تكون محلية أو دولية مثل (ASTM) و ، وغيرهما) تحدد مجالات استخدامه [5] · ل وبالرجوع الى الادبيات نجد العديد من الدراسات التي تتاولت عملية تحوير مواصفات الاسفلت بطرائق مختلفة ومنها ما قام به Mohammed الفحوصات الريولوجية للإسفلت فضلاً عن فحص ثباتية الخزن بدرجات حرارية عالية واستتتج أن اضافة البوليمر الى الاسفلت تودي الى لى

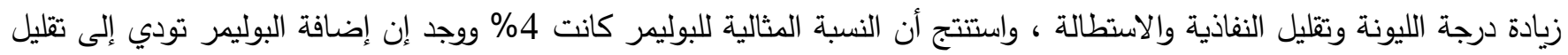
الحساسية الحرارية. وفي بحثاً اخر [7] تم تحوير الخواص الريولوجية لإسفلت القيارة وباستعمال تقنية المايكروويف وعند طاقتي (180 و 360) واط على التوالي وباستعمال نسب وزنية مختلفة من مطاط الإطارات المعاد وباستخدام حفاز كلوريد الألمنيوم اللامائي وبنسب وزنية مختلفة مع نسب وزنية مختلفة من الكبريت وبعدها تمت دراسة الخواص الريولوجية من حيث الاستطالة، والنفاذية، ودرجة الليونة، ودليل 


\section{Journal of Education and Science (ISSN 1812-125X), Vol: 29, No: 3, 2020 (85-101)}

الاختراق. وأخذ أفضل انموذج من حيث الخواص الريولوجية والمطابق لمواصفات هيئة الطرق والجسور العراقية كاسفلت تبليط وأجري عليه اختبار مارشال وكانت النتيجة جيدة من حيث قيم الزحف والاستقرارية.

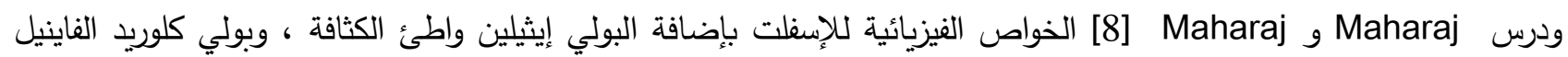
(PVC)، وزيت المحركات المستعمل • إذ بينت النتائج أنه بازدياد تركيز زيت المحركات المستعمل بنسب (0-30\%) حصلت زيادة في صفة مقاومة تشققات الإجهاد ، ورافقه انخفاضٌ في مقاومة تشكل الأخاديد في الثارع.

وقام Dekhli [9] وجماعته بدراسة السلوك الريولوجي للإسفلت المحوَّ بإضافة إيثلين فاينل أسيتيت (EVA)، أعطت الدراسة نماذج إسفلتية ذات مواصفات ريولوجية بالإمكان استعمالها في مجال التبليط ـ إذ كان هنالك تغير إيجابي واضح في القياسات (درجة الليونة ، النفاذية ، الحساسية لدرجة الحرارة) •

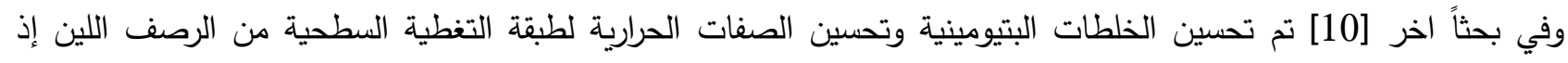

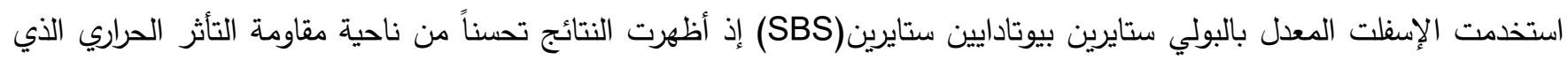
تتعرض له. كما تمكن Thakre وجماعته [11] من تحوير الصفات الريولوجية للأسفلت بإضافة إيثلين فاينل أسيتيت (EVA) ، وفتات المطاط ـ وأعطت الدراسة نماذج إسفلتية أكثر مقاومةً للإجهاد(fatigue)، والتشققات الحرارية ، والتعفن (Rutting) ، والحساسية لدرجة الحرارة . وبالإمكان استعمالها في مجال التبليط . وتمكن Gama وجماعته [12] من دراسة الخواص الريولوجية للإسفلت المحوَّر بالبوليمرات من نوع (إيثيلين ميثايل اكريليتكلاسيديل ميثاكريليت)(Ethylene Methyl Acrylate - Glycidy Methacrylate (GMA-EMA. والبولي إيثلين عالي الكثافة(HDPE) Acid Polyphosphoric إضافة الى متعدد حامض الفسفوريك وكان لاستخدام هذه البوليمرات معاً تأثيراً واضحاً في تحسين خواص المرونة للإسفلت المحوَّر مع أداء عالِ في العمل (PPA116\%) في درجات الحرارة العالية والمتوسطة. فضلاً عن ذلك ، قام كل من Imanisab Shirini و13] بدراسة الخواص الريولوجية للإسفلت المسامي المحوَّر بالمطاط ، بالمقارنة مع خلائط الأسفلت المسامي المحوَّ بال(SBS) . ووجد أن إضافة (10\%) من فتات المطاط يؤدي إلى تحسين أداء الإسفلت

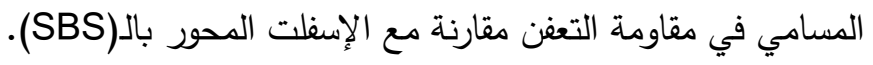

وفي بحثاً اخر [14] تم تحسين المقاومة الحرارية وتقليل تأثير الاهتزازات في الخلطة الاسفلتية باستخدام مفروم الإطارات المستهلكة والبوليمرات إذ تمت إضافة مادة النوفولاك بنسب مختلفة من وزن إسفلت الدورة مع إضافة مفروم الاطارات المستهلكة بنسب مختلفة من وزن اسفلت الدورة وقد كانت النتائج بعد إجراء الفحوصات جيدة من ناحية زيادة قوة الثبات وانخفاض في قيمة الاختراق والسحب للإسنفلت المحسن.

وتمكن Kareem [15 من دراسة تأثير تعديل البوليمرات على خواص الأسفلت الرابط باستخدام نوعين من البوليمرات، ستايرين بيوتادايين ستايرين (SBS) وإيثايل فانيل أسيتيت (EVA) بنسب وزنية مختلفة ومن ثم تقييم تأثير هذه التعديلات على خواص

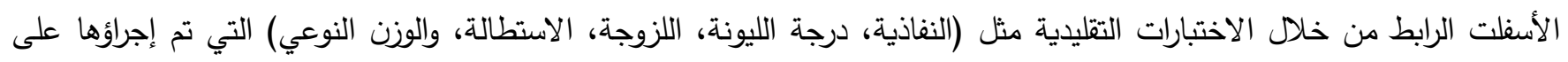
الأسفلت الرابط المعدل والطبيعي ووجد بأن خواص الأسفلت الرابط المعدل كانت أفضل بكثير بالمقارنة مع الإسفلت الطبيعي. 
وقد استطاع nekhoroshev [16] وجماعته من اضافة مادة البولي بروبيلين اتاكتك (APP) الى الأسفلت التي تم الحصول عليها عن طريق البلمرة ، حيث تمت عملية الاضافة عند 140ْم حيث أدى ذلك الى اكسدة APP مما أدى الى انتاج أسفلت مقاوم للتدهور الحراري والتقادم والالتصاق العالي. وفي بحثاً اخر [17] تم تحوير الخواص الريولوجية لإسفلت القيارة باستخدام الموجات فوق الصوتية وباستخدام مطاط الإطارات المعاد بنسب وزنية مختلفة وبوجود حفاز كلوريد الألمنيوم اللامائي بنسب وزنيه مختلفة مع نسب وزنية مختلفة من الكبريت ومن ثم الإن دراسة الخواص الريولوجية الناتجة من حيث الاستطالة، والنفاذية، درجة الليونة، ودليل الاختراق. وأخذ أفضل نموذج من حيث الخواص الريولوجية والمطابق لمواصفات هيئة الطرق والجسور العراقية كإسفلت تبليط وأجريت عليه اختبار مارشال وكانت النتيجة ممتازة من جهة

$$
\text { قيم الزحف والاستقرارية . }
$$

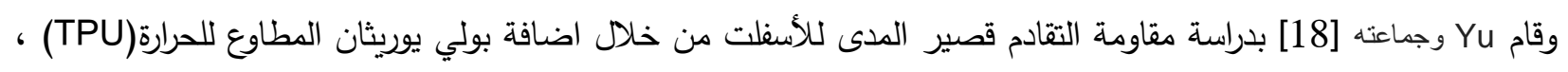
حيث وجذَ أن اضافة هذا البوليمر يعمل على تحسين الخواص الحرارية ، واستقرار التخزين في درجات الحرارة العالية، وايضاً أثثتت نتائج التحليل الطيفي للأشعة تحت الحمراء تحسن في مقاومة التقادم الزمني للأسفلت.

وفي بحثاً اخر [19] تم تحوير الخواص الريولوجية للإسفلت باستعمال الزيوت المستهلكة والأكسدة الهوائية وقد تم الحصول

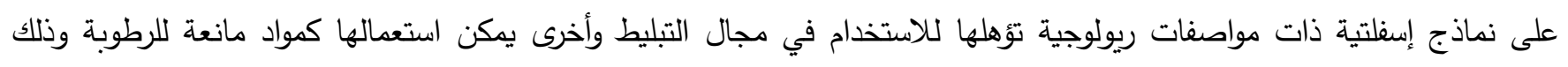
اعتمادا على الفحوصات التي تم إجراؤها مثل الاستطالة، النفاذية ، درجة الليونة ، نسبة الاسفلتين و دليل الاختراق.

1. إسفلت الدورة الخام (الإسفلت الأساس) المنتج في مصفى الدورة يتميز بالمواصفات الموضحة في جدول(1). جدول (1): الخواص الفيزيائية العامة لإسفلت الدوة (الأسفلت الأساس)

\begin{tabular}{|c|c|}
\hline 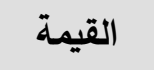 & الخواص الفيزيائية العامة \\
\hline 50 & درجة الليونة (مº) \\
\hline 44.6 & النفاذية (100غم، 5 ثا ، 25مº) \\
\hline $100+$ & الاستطالة (سم، 25مم) \\
\hline-1.448 & دليل الاختراق(PI) \\
\hline 17.81 & نسبة الأسفلتين (\%) \\
\hline
\end{tabular}

2. الكبريت إذ تمَ الحصول عليه من الشركة العامة لكبريت المشراق.

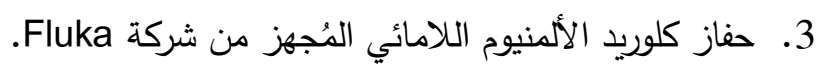
4. بولي يوريثان المطاوع للحرارة (المستخرج من المخلفات الداخلية للسيارات) 


\section{Instruments Used}

Ductility Measurement Apparatus
ثانيا: الأحهزة المستخدمة

$$
\text { 1- جهاز قياس الاستطالة }
$$

يعمل هذا الجهاز على قياس المسافة التي تستطيل بها المواد الأسفلتية عند تعرضها لتأثير سحبٍ وبسرعةٍ ثابتة، إلى أن ينقطع الأنموذج الأسفلتي. والجهاز ذو منشأ صيني من نوع(YUFENG).

Penetrometer

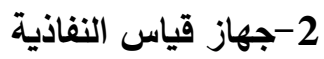

يعمل الجهاز على فحص نفاذية المواد البتيومينيـة الصلبـة وشبـه الصلبـة. فالنفاذيـة هي مقيساس لصلابـة الأسفلت. والجهاز ذو منشأ صيني نوع(YUFENG).

Ring and Ball Apparatus

$$
\text { 3-جهاز قياس درجة الليونة }
$$

يعدل هـذا الجهـاز على قيـاس درجة الليونة للمواد الأسفلتية التي تتراوح ليونتها ما بين (30-200)خُ، ودرجة الليونة هي الدرجة الحرارية التي ينزل عندها الأنموذج الأسفلتي مسافة (2.54)سم عند تسخينه بسرعة (5م/ دقيقة) مع تجنب التسخين السريع Fast) .heating)

\section{Asphalt Treatment Apparatus}

4

$$
\text { يتكون الجهاز من الأجزاء الآتية : }
$$

1. دورق زجاجي كبير بحجم 200مل ثلاثي الفتحات (Angled Three Neck Round Bottom Flask).

2. محرار مُثبت على أحد الفتحات الجانبية.

3. مُشخن كهربائي (Heating Mantle).

4. محرك ميكانيكي يُثبت على الفتحة الوسطى للدورق .

5. حامل حديدي مُزود بماسك لتثبيت الدورق.

والثكل الآتي(1) يوضح الجهاز الذي استعمل في عملية معالجة الإسفلت

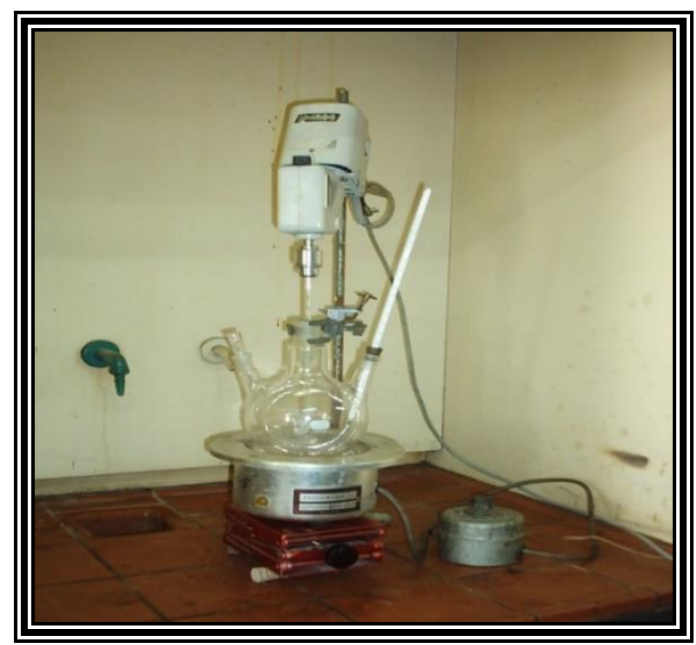

الشكل(1): جهاز معالجة الإسفلت 


\section{Journal of Education and Science (ISSN 1812-125X), Vol: 29, No: 3, 2020 (85-101)}

Microwave Oven

5

استخدم فرن المايكروويف ذو المنشأ الألماني من نوع(Tokiwa) وبقدرة (900 واط) وترددMHz (2450) في عملية تحضير النماذج الأسفلتية المحورة.

\section{Electrical shaker}

$$
6
$$

Hamber Ggo Shaker استخدم هذا الجهاز لرج محلول (أسفلت -هكسان اعتيادي) لغرض فصل الأسفلت، وهو من نوع .Germany

\section{Marshall Testing Apparatus}

- 7 - (خهاز اختبار مارشال

هذا الفحص يعطي دلالة عن مدى ملائمة الأسفلت للتبليط ، والجهاز ذو منشأ إنكليزي نوع (WYKEHAM FARRANCE).

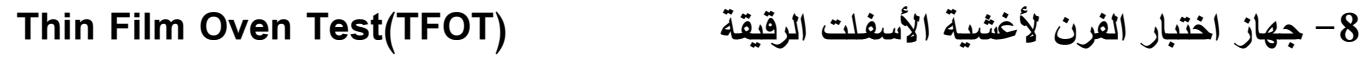

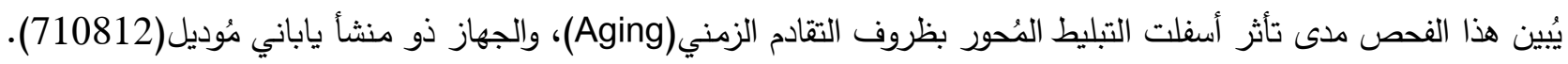

ثالثاً :- طريقة العمل: (Experimental Method)

1 - تحـوير الخـواص الريولوجيـة للإِــفلت بالمعالجـة الكيميائيـة المحفـزة مـع بـولي يوريثـان المطــاوع للحـرارة باسـتخدام تقنيـة المايكروويف:

وضـع وزن 250 غم دن إسفلت الدورة في جهاز معالجة المادة الإسفلتية (الموضـح في الفقرة 4 من الاجهزة المستخدمة)، وسُخن إلى درجـة حرارة (100)مْ، بعدها تمت إضـافة (0.5)\% من بولي يوريثان المطاوع للحرارة، ومن ثم إضـافة كلوريد الألمنيوم

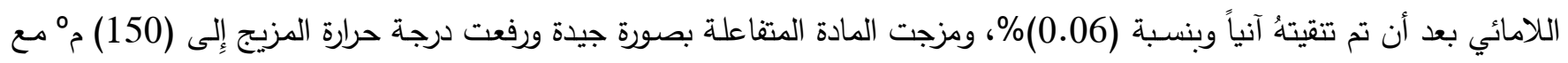

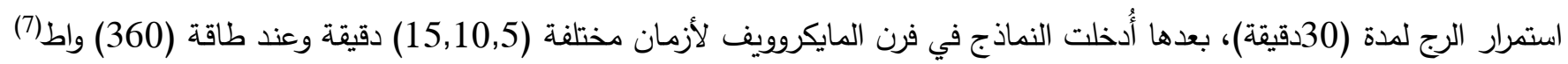

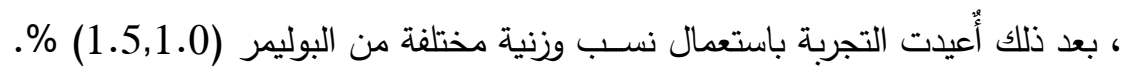
تم إجراء قياسـات الاستطالة [20]، والنفاذية [21]، ودرجـة الليونـة [22] ودليل الاختراق [23] ، والنتائج مدرجة في الجدول

2- تحوير الخواص الريولوجية للإسفلت بالمعالجـة الكيميائية المحفزة بوجود الكبريت مـع بولي يوريثان المطاوع للحرارة باستخدام تقنية المايكروويف :

وضع وزن 250 غم من إسفلت الدورة في جهاز معالجة المادة الإسفلتية (الموضح في الفقرة 4 من الاجهزة المستخدمة)، وسُخن إلى درجة حرارة (100)مْه بعدها تمت إضـافة (0.5)\% من البولي يوريثان المطاوع للحرارة، ومن ثم إضـافة (1)\% كبريت وباستخدام

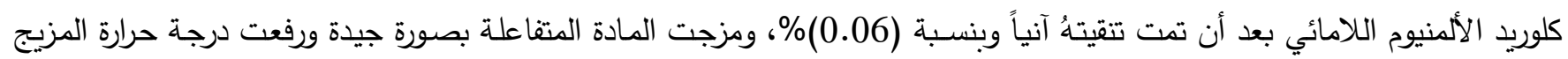

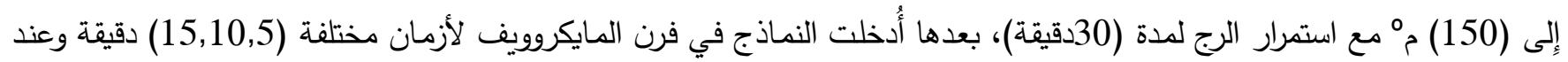

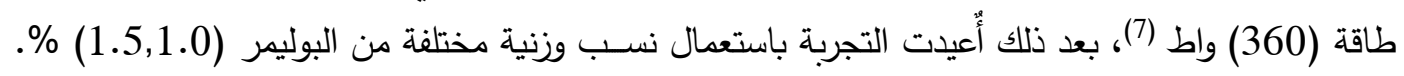

تم إجراء قياسات الاستطالة [20]، والنفاذية [21]، ودرجة الليونة [22] ودليل الاختراق [23] ، والنتائج مدرجة في الجدول (3). 


\section{Journal of Education and Science (ISSN 1812-125X), Vol: 29, No: 3, 2020 (85-101)}

3- تحوير الخـواص الريولوجيـة للإسـفلت بالمعالجـة الكيميائيـة المحفزة مـع بـولي يوريثان المطاوع للحرارة بـدون استخدام تقنيـة المايكروويف :

وضـع وزن 250 غم من إسفلت الدورة في جهاز معالجة المادة الإسفلتية (الموضـح في الفقرة 4 من الاجهزة المستخدمة)، وسُخن إلى درجـة حرارة (100)مْ؛ بعدها تمـت إضـافة (0.5)\% من بولي يوريثان المطاوع للحرارة، ومن ثم إضـافة كلوريد الألمنيوم

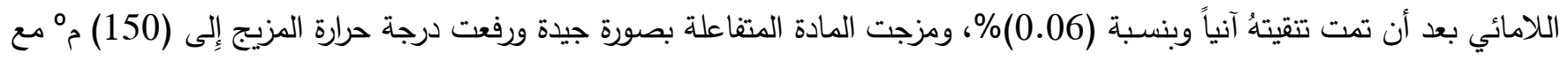

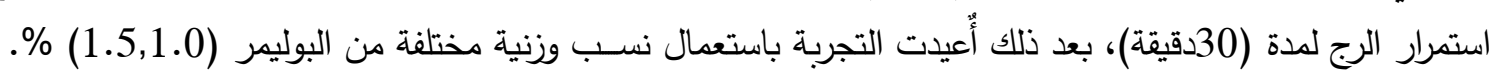

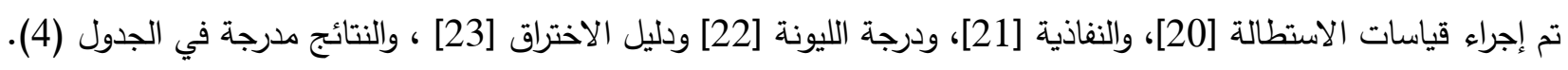

\section{Asphaltene separation} 4

وضعت كمية من إسفلت الدورة الاصل(1غم ) في داخل وعاء مخروطي ذي غطاء مُحكم وأُضيف لها (40 مل) هكسان اعتيادي بنسبة (40:1) ( وزن : حجم) ورج المحلول لمـدة(3 ساعات) باستخدام جهاز الرج الكهربائي بدرجة حرارة الغرفة وبعدها رُشَح المحلول وغُسل

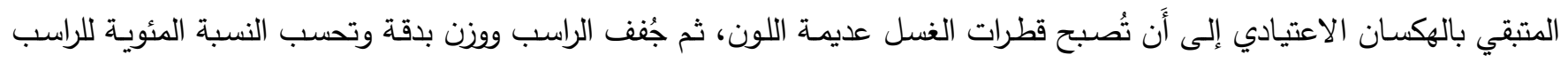
(الإسفلتين), وبعدها طبقت عملية فصل الاسفلتين على جميع النماذج المحضرة.

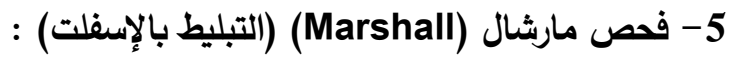

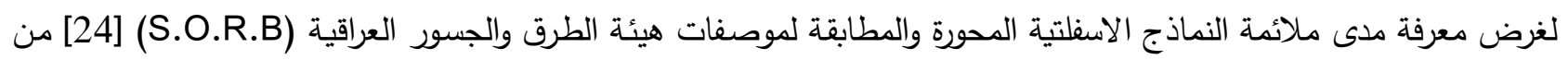
خلال حساب الاستقرارية والزحف للنماذج لكل من الأساس والمحور وحسب طريقة معهد الإسفلت الأمريكي [25] ، والنتائج مدرجة

$$
\text { في الجدول (4). }
$$

6- اختبار الفرن لاغثية الاسفلت الرقيقة (Thin Film Oven Test) :

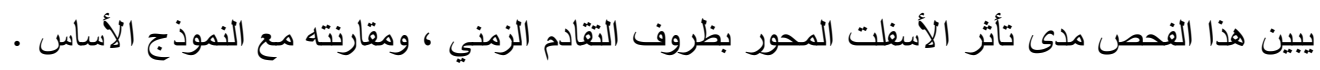
وقد اجري الفحص وفق المواصفات القياسية الامريكية (2002-1754 - ASTM [26] ، كما هو موضح في الجدول (6) .

\section{(Results and discussion) : النتائج والمناقشة}

1 - تحـوير الخـواص الريولوجيـة للإسـفلت بالمعالجـة الكيميائيـة المحفـزة مـع بـولي يوريثـان المطـاوع للحـرارة باسـتخدام تقنيـة المايكروويف: تهدف هذهِ الدراسة إلى تحوير اسفلت الدورة بالبولي يوريثان المطاوع للحرارة من أجل تحسين مواصفاته لجعلها تتلائم من استخدامه كأسفلت للتبليط، ويمكن تحقيق ذلك بأساليب مختلفة، الأول يعتمد على ربط جزيئة البوليمر مع جزيئة اسفلت الدورة وباستخدام(0.06\%) من كلوريد الألمنيوم اللامائي كعامل مُحفز لهذٍِِ العملية وعند طاقة 360 واط حسب الدراسات السابقة [7] ، ويُبين الجدول (2) نتائج هذه 
الجدول (2): المواصفات الريولوجية لإسفلت الدورة المُحوّر مع نسب مختلفة من بولي يوريثان المطاوع للحرارة، وبوجود نسبة (\%0606) من حفاز كلوريد الألمنيوم اللامائي عند طاقة (360) واط وبأزمان مختلفة.

\begin{tabular}{|c|c|c|c|c|c|c|c|}
\hline الاسفلتين & $\begin{array}{c}\text { الاخترلق } \\
\text { (PI) }\end{array}$ & الليونة & 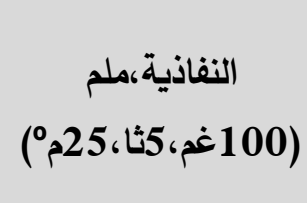 & $\begin{array}{c}\text { الاستطالة } \\
\text { (25،cm) }\end{array}$ & $\begin{array}{l}\text { الزمن } \\
\text { (min) }\end{array}$ & 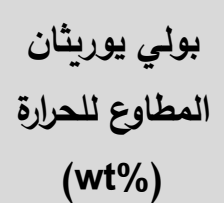 & الانموذج \\
\hline 17.81 & -1.448 & 50 & 44.6 & +100 & 0 & 0 & $A s_{0}$ \\
\hline 21.25 & 0.197 & 58 & 41.8 & +100 & 5 & 0.5 & $A s_{1}$ \\
\hline 23.10 & 0.511 & 60 & 40.3 & +100 & 5 & 1.0 & $\mathrm{As}_{2}$ \\
\hline 23.83 & 1.037 & 63.5 & 38.1 & 52 & 5 & 1.5 & $\mathrm{As}_{3}$ \\
\hline 21.61 & -0.850 & 54 & 43.2 & +100 & 10 & 0.5 & $\mathrm{As}_{4}$ \\
\hline 23.49 & 0.474 & 60 & 39.7 & 84 & 10 & 1.0 & $\mathrm{As}_{5}$ \\
\hline 25.03 & 0.826 & 62 & 39.1 & 65 & 10 & 1.5 & $\mathrm{As}_{6}$ \\
\hline 20.01 & 0.405 & 59 & 41.9 & +100 & 15 & 0.5 & $\mathrm{As}_{7}$ \\
\hline 20.59 & 0.791 & 61.5 & 40.1 & 89 & 15 & 1.0 & $A s_{8}$ \\
\hline 24.48 & 0.964 & 63 & 38.3 & 67 & 15 & 1.5 & $\mathrm{As}_{9}$ \\
\hline
\end{tabular}

As يتضح لنا من الجدول (2)، أنه بزيادة نسبة بولي يوريثان المطاوع للحرارة تقل قيم النفاذية وتزداد قيم الليونة، لذلك تفضل القيم المنخفضة من البوليمر، وهذا ما أكدتهُ الدراسات الحديثة التي تبين أنَ النسب الواطئة من البوليمر هي النسب المفضلة في إجراء التحويرات الريولوجية، حيث يكون طور البوليمر منتشراً في نظام طور الإسفلت المستمر [27]. اما بالنسبة للإسفلتين فنلحظ من الجدول (2) أن بزيادة نسبة البوليمر المضاف مع زيادة زمن التعرض لأشعة المايكروويف تزداد نسبة الإسفلتين مقارنة مع أسفلت الاساس اذ ان زيادة نسبة البوليمر المضاف ادت الى زيادة الوزن الجزيئي للإسفلت بسبب ارتباط الجزيئات البوليمرية ذات الاوزان الجزيئية العالية بالإسفلت، أما فيما يتعلق بالحساسية الحرارية للنماذج التي تم تحضيرها إذ تم حساب دليل الاختراق (PI) فظهرَ أن جميع النتائج المحصلة عليها كانت ضمن الحدود (+2_2-) وهذا يثبت على أن النظام الإسفلتي بأنه من نوع)[23] (Sol_Gel_Asphalt) 
2- تحوير الخواص الريولوجية للإسفلت بالمعالجة الكيميائية المحفزة بوجود الكبريت مع بولي يوريثان المطاوع للحرارة باستخام

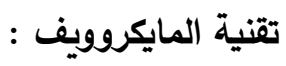
يعتمد هذا الأسلوب من التحوير على ربط جزيئة البولي يوريثان المطاوع للحرارة مع جزيئة الإسفلت بوجود الكبريت واستخدام كلوريد الألمنيوم اللامائي كعامل مُحفز لهذهِ العملية، ويُبين الجدول(3) نتائج هذه المعالجة.

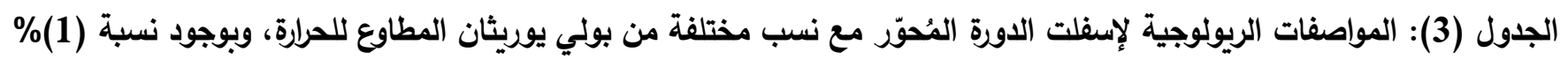
كبريت ونسبة (0.06\%) من حفاز كلوريد الألمنيوم اللامائي وغند طاقة (360) واط وبأزمان مختلفة.

\begin{tabular}{|c|c|c|c|c|c|c|c|}
\hline الاسفلتين & $\begin{array}{c}\text { الاخترلق } \\
\text { (PI) }\end{array}$ & الليونة & (النفاذية،ملم 100 غم،5ثا، 25م') & الاستطالة & $\begin{array}{l}\text { الزمن } \\
\text { (min) }\end{array}$ & $\begin{array}{c}\text { بولي يوريثان المطاوع للحرارة } \\
\text { (wt\%) }\end{array}$ & رقم \\
\hline 17.81 & -1.448 & 50 & 44.6 & +100 & 0 & 0 & $\mathrm{As}_{0}$ \\
\hline 21.40 & 0.166 & 58 & 41.3 & +100 & 5 & 0.5 & $A s_{10}$ \\
\hline 25.92 & 0.295 & 58.5 & 41.7 & +100 & 5 & 1.0 & $\mathrm{As}_{11}$ \\
\hline 28.20 & 0.585 & 61 & 38.2 & 70 & 5 & 1.5 & $A s_{12}$ \\
\hline 21.72 & 0.107 & 58 & 40.2 & +100 & 10 & 0.5 & $\mathrm{As}_{13}$ \\
\hline 26.46 & 0.478 & 59.5 & 41.4 & +100 & 10 & 1.0 & $\mathrm{As}_{14}$ \\
\hline 29.10 & 0.748 & 62 & 37.8 & 64 & 10 & 1.5 & $\mathrm{As}_{15}$ \\
\hline 21.56 & 0.050 & 57 & 42.9 & +100 & 15 & 0.5 & $A s_{16}$ \\
\hline 27.81 & 0.398 & 59 & 41.8 & +100 & 15 & 1.0 & $\mathrm{As}_{17}$ \\
\hline 28.50 & 0.585 & 60.3 & 40.6 & 95 & 15 & 1.5 & $A s_{18}$ \\
\hline
\end{tabular}

As 0 
يلاحظ من الجدول (3) إن إضافة الكبريت إلى النماذج الإسفلتية المُحوّرة بواسطة بولي يوريثان المطاوع للحرارة يعمل على زيادة

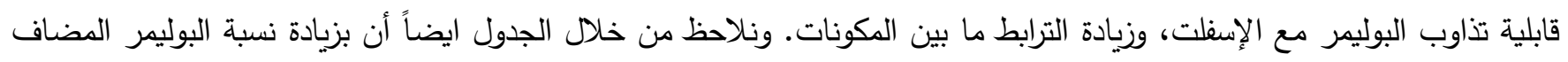

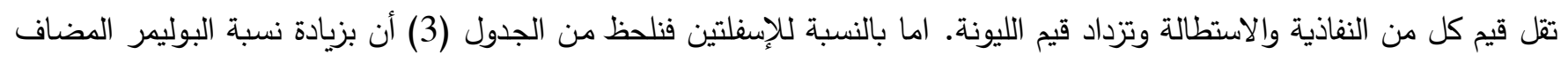

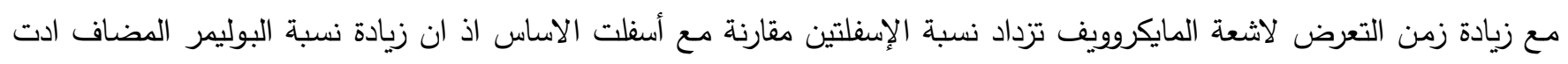
الى زيادة الوزن الجزيئي للإسفلت بسبب ارتباط الجزيئات البوليمرية ذات الاوزان الجزيئية العالية بالإسفلت، أما فيما يتعلق بالحساسية

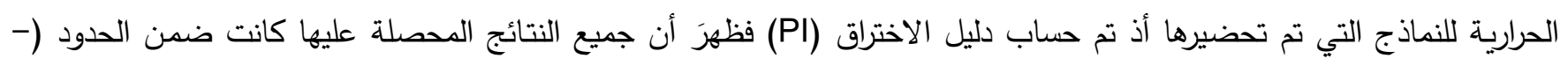

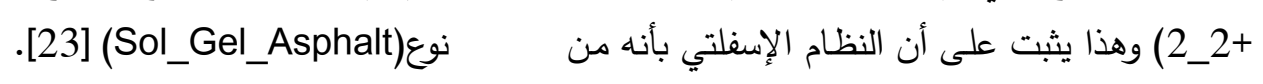

3- تحوير الخواص الريولوجية للإسفلت بالمعالجة الكيميائية المحفزة مع بولي يوريثان المطاوع للحرارة بدون استخدام تقنية المايكروويف : يعتمد هذا الأسلوب من التحوير على ربط جزيئة البولي يوريثان المطاوع للحرارة مع جزيئة الإسفلت باستخدام كلوريد الألمنيوم التلامائي كعامل مُحفز لعملية التحوير بدون فرن المايكروويف ، ويُبين الجدول(4) نتائج هذه المعالجة.

الجدول (4): المواصفات الريولوجية لإسفلت الدورة المُحوّر مع نسب مختلفة من بولي يوريثان المطاوع للحراة ، وبوجود

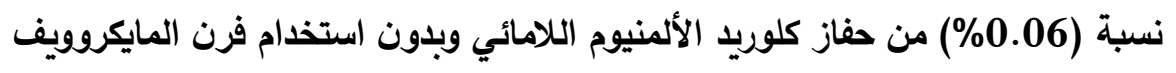

\begin{tabular}{|c|c|c|c|c|c|c|c|}
\hline الاسفلتين & $\begin{array}{c}\text { دليل } \\
\text { الاختراق } \\
\text { (PI) }\end{array}$ & الليونة & (100 (النفاذية،ملم، 5ثا، 25مم) & $\begin{array}{c}\text { الاستطالة } \\
\text { (25،cm) }\end{array}$ & $\begin{array}{l}\text { الزمن } \\
\text { (min) }\end{array}$ & $\begin{array}{c}\text { المطاوع يوريثان } \\
\text { (wt\%) }\end{array}$ & الانموذج \\
\hline 17.81 & -1.448 & 50 & 44.6 & +100 & 0 & 0 & $\mathrm{As}_{0}$ \\
\hline 18.51 & 0.634 & 60.5 & 40.8 & +100 & 30 & 0.5 & $\mathrm{As}_{19}$ \\
\hline 19.24 & 1.127 & 64 & 38.1 & 75 & 30 & 1.0 & $\mathrm{As}_{20}$ \\
\hline 19.83 & 1.303 & 66 & 35.2 & 46 & 30 & 1.5 & $\mathrm{As}_{21}$ \\
\hline
\end{tabular}

As 0

يلاحظ من الجدول (4) ان زيادة نسبة البوليمر سوف يؤدي ذلك الى تدهور المواصفات الريولوجية، ويلاحظ من خلال الجدول ايضاً

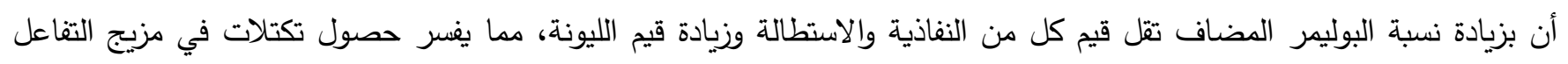
نتيجة ارتباط عدد كبير من البوليمر مع جزيئات الاسفلت بمساعدة كلوريد الالمنيوم اللامائي • 


\section{Journal of Education and Science (ISSN 1812-125X), Vol: 29, No: 3, 2020 (85-101)}

كما يلاحظ من الجدول (4) ان النتائج المستحصلة بدون استخدام المايكروويف كانت اقل كفاءة مما في حال استخدام المايكروويف، كما أن استعمال أشعة المايكروويف في التسخين يخفض كمية الغازات المنبعثة أثناء المعالجة مما يؤدي إلى تقليل التلوث البيئي، كانت النسبة 0.5\% من البولي يوريثان المطاوع للحرارة هي النسبة الأفضل في تحوير الخواص الريولوجية لإسفلت الدورة، إذ لوحظ أن زيادة نسبة البوليمر المُضافة للأسفلت تُؤدي إلى تدهور المواصفات الريولوجية، من الصعوبة جداً أن تكون هناك علاقة ثابتة تربط بين المتغيرات التي تطرأ على الأنظمة الإسفلتية أثناء معالجتها بالبوليمرات، وذلك لأن طبيعة التفاعل معقدة جداً.

4- فحص مارشال (Marshall) (التبليط بالأسفلت): لغرض معرفة مدى مُلاءمة النماذج المحورة بتقنية المايكروويف (As $)$ لأغراض التبليط تم إجراء فحص مارشال (التبليط بالأسفلت) للنماذج التي أظهرت كانت مواصفاتها مُطابقة لمواصفات هيئة الطرق والجسور العراقية (S.O.R.B) لعام 2001 كأسفلت تبليط [24]، ولإِسفلت الأساس أيضاً، إذ تم الفحص كما هو مُبين في الجدول (5) •

الجدول (5): يوضح قيم الاستقرية والزحف لإسفلت الدورة المُحوّر والمقارنة مع إسفلت الدورة الأساس، ومواصفات هيئة الطرق والجسور (S.O.R.B).

\begin{tabular}{|c|c|c|c|}
\hline $\begin{array}{l}\text { الزحف } \\
\text { (mm) }\end{array}$ & $\begin{array}{c}\text { الإستقرارية } \\
\text { (KN) }\end{array}$ & نسبة الإسفلت (\%) المضاف إلى الركام & رقم الانموذج الإسفلتي \\
\hline 5.1 & 11.3 & \multirow[b]{2}{*}{4.5} & $\mathrm{As}_{0}$ \\
\hline 3.2 & 21.3 & & $A s_{7}$ \\
\hline $4-2$ & $\begin{array}{c}7 \\
\text { Minimum }\end{array}$ & $5.5-3$ & $\begin{array}{c}\text { مواصفات } \\
\text { (S.O.R.B) }\end{array}$ \\
\hline
\end{tabular}

يتضح من الجدول (5) أن قيم الاستقرارية للإسفلت الأساس كانت ضمن مواصفات هيئة الطرق والجسور العراقية (S.O.R.B) كإسفلت تبليط [24]، ولكن قيم الاستقرارية للنموذج الإسفلتية المحورة كانت أفضل بكثير من قيمة الاستقرارية للإسفلت الأساس إذ يلاحظ ارتقاع قيمتها وهذا مؤشر جيد على قدرة مقاومة التبليط للتشوه الناجم عن تعرض الطريق للأحمال المُتكررة لوسائط النقل . ولكن قيمة الزحف للإسفلت الأساس كانت خارج مواصفات هيئة الطرق والجسور العراقية (S.O.R.B) كأسفلت تبليط وهذا ما يفسر تعرض الشوارع المبلطة بأسفلت الدورة دون معالجة الى التَََكُل والتخدد مما يسبب تبعات اقتصادية سيئة لدى استخدامه في التبليط من دون اجراء المعالجات والتحويرات اللازمة للهُ.

في حين أن قيم الزحف للنموذج الأسفلتي المحور كان افضل بكثير من قيمة الزحف للأسفلت الأساس إذ يلاحظ انخفاض قيمتها

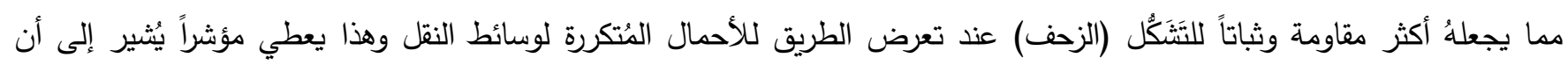

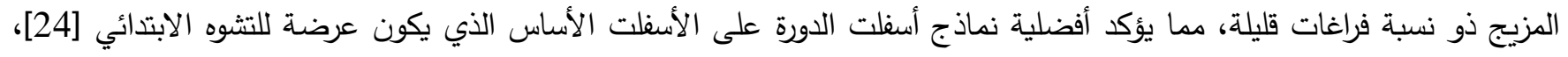
وهذا ما يُعسر تعرض الكثير من الطرق المُبلطة اليوم بهذا النوع من الإسفلت للزحف والتخدد وعدم الاستقرارية. 
5- اختبار الفرن لاغشية الاسفلت الرقيقة (Thin Film Oven Test)

تم اجراء الدراسة هذه على كل من النموذج الأساس والمحور ، والنتائج موضحة في الجدول (6).

الجدول (6): تفيير الموصفات الريولوجية لأسفلت الدورة المحور واسفلت الدورة الأساس بعد اخضاعهما لفحص الفرن لأغشية الاسفلت الرقيقة (TFOT)

\begin{tabular}{|c|c|c|c|c|c|}
\hline الفرق & بعد الفحص & قالفحص & الصفات الريولوجية & وصف النموذج & رقم النموذج \\
\hline $\begin{array}{c}3.3 \\
3\end{array}$ & $\begin{array}{c}+100 \\
41.3 \\
53 \\
-0.909 \\
0.05\end{array}$ & $\begin{array}{c}+100 \\
44.6 \\
50 \\
-1.448 \\
-----\end{array}$ & 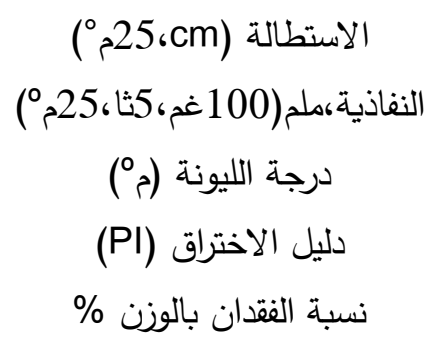 & الأساس & $\mathrm{As}_{0}$ \\
\hline $\begin{array}{c}1.8 \\
2\end{array}$ & $\begin{array}{c}+100 \\
40.1 \\
61 \\
0.695 \\
0.029\end{array}$ & $\begin{array}{c}+100 \\
41.9 \\
59 \\
0.409 \\
-----\end{array}$ & 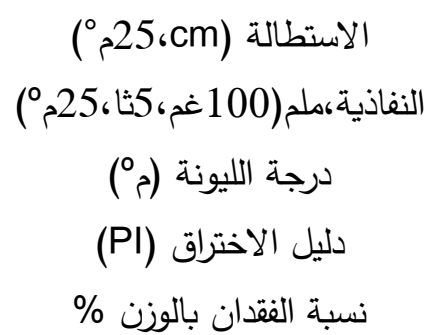 & المحور & $\mathrm{As}_{7}$ \\
\hline
\end{tabular}

يلاحظ من الجدول (6) ان نسبة تأثر الاسفلت المحور بظروف التقادم الزمني من درجة حرارة و اوكسجين بشكل عام تكون قليلة هذا

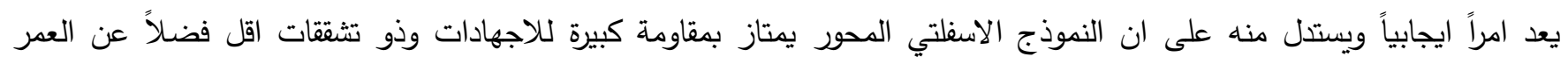
التشغيلي الطويل ، وان التأثر المحدود للنموذج الاسفلتي المحور بظروف التقادم الزمني يعزى الى ما يتمتع به البولي يوريثان المطاوع للحرارة الذي يعمل على تحسين الخواص الميكانيكية والمتمثلة بزيادة متانة النموذج الاسفلتي وتحمله للاجهادات وتقليل التكسير الحراري وزيادة مقاومته لتكوين الاخاديد [28,29].

وبالرجوع إلى الجداول السابقة (2) و(3) و(4) نجد أن بعض النماذج تميزت بدرجات ليونة عالية وقيم نفاذية واستطالة واطئة تؤهل استخدامها في إنتاج الماستك (Mastic) المستخدم كمادة عازلة للرطوبة (Water Proofing)، ونماذج أُخرى بالإمكان استخدامها كإسفلت يُستعمل في التسطيح بعد إخضاعها للاختبارات الهندسية. 
والجدول (7) يبـين قيم النفاذيـة والاستطالة ودرجـة الليونـة للإِـفلت المستعمل كماستك عـازل للرطوبـة وحسب المواصـفات القياسية الأمريكية (8STM(D491-AST المعتمدة عالمياً [30]. الجدول (7): المواصفات القياسية الامريكية (881-ASTM(D491) للإسفلت المستعمل لإنتاج الماستك.

\begin{tabular}{|c|c|c|}
\hline الحد الأعلى & الحد الأدنى & القياسات الريولوجية \\
\hline 65 & 54 & درجة الليونة (مº) \\
\hline 40 & 20 & النفاذية، ملم (100 غم، 5ثا، 25مº \\
\hline . & 15 & الاستطالة (سم، 25مº \\
\hline
\end{tabular}

أما المواصفات القياسية العراقية للإِففلت المستعمل لأغراض التسطيح فيُينها الجدول (8) [31]. الجدول (8): المواصفات القياسية العراقية للإسفلت المستعل في التسطيح.

\begin{tabular}{|c|c|c|}
\hline الصد الأعلى & الحد الأدنى & القياسات الريولوجية \\
\hline 66 & 57 & درجة الليونة (مº) \\
\hline 40 & 18 & النفاذية، ملم (100غر، 5ثا، 25مº \\
\hline. & 10 & الاستطالة (سم، 25مº \\
\hline
\end{tabular}

والجدول (9) يبين الخواص الريولوجية لإسفلت التبليط حسب مواصفات هيئة الطرق والجسور العراقية (S.O.B.R) لعام 2001 [24]. الجدول (9): الخواص الريولوجية للاسفلت المستعمل في التبليط.

\begin{tabular}{|c|c|c|}
\hline الحد الأعلى & الحد الأدنى & المواصفات الريولوجية \\
\hline . & 100 & الاستطالة (cm،cmمº) \\
\hline 50 & 40 & النفاذية ملم، (100 غم.5ثا.25مم) \\
\hline 60 & 54 & درجة الليونة (مم) \\
\hline
\end{tabular}


(a إن استخدام تقنية التسخين بالمايكروويف (Microwave) في التسخين يخفض كمية الغازات المنبعثة أثناء المعالجة مما يؤدي إلى التقليل التلوث البيئي.

b ) إن نسبة (0.5)\% من البولي يوريثان المطاوع للحرارة تمثل النسبة الأمثل في تحوير الخواص الريولوجية لأسفلت الدورة. (c سابقة][32].

(d من الصعوبة أن نجد علاقة ثابتة تربط بين المتغيرات التي تطرأ على الأنظمة الأسفلتية أثثاء معالجتها بالبوليمرات وذلك لأن طبيعة التفاعل الذي يحدث يكون معقدة جداً.

e e إن نجاح بعض العينات المُحورة في اختبارات مارشال والتقادم تعد مؤشراً إيجابياً على إمكانية استخدامه في التبليط إذ إن قيم الزحف والاستقرارية للأسفلت المُحور كانت أفضل بكثير من قيم الزحف والإستقرارية للأسفلت الأساس.

يتقدم الباحثون بالثكر والتقدير لقسم الكيمياء في كلية التربية للعلوم الصرفة - جامعة الموصل وكلية الهندة جامعة الموصل لتقديم التسهيلات اللازمة لاكمال هذا البحث .

\section{المصادر}

1- Henglein, F.A., "Chemical Technology", Pergamon Press, $\quad$ London,pp.811-812,(1969).

2- Hobson, G.D. "Modern Petroleum Technology".4 $4^{\text {th }}$ ed., Ltd. Britain, $\quad$ pp.804-806,(1973).

3- Lesueur D., "The Colloidal Structure of Bitumen, Consequences on the Rheology and on The Mechanisms of Modification", Advances in Colloid and Interface Science, pp. 145, 28-42,(2009).

4- "Introduction to Asphalt", Asphalt institute manual series No. 5 (MS-5) P.2, 9-11 14, 61, (2001).

5- Asphalt Institute Inc. and European Bitumen Association, Eurobitume, The Bitumen Industry, A Global Perspective, $3^{\text {rd }}$ ed., USA (2015). 
6- Dhirar T.M., Zaid H. H., International Journal of Enhanced Research in Science Technology \& Engineering, Vol. 3 Issue 9, pp: (114-121), (2014).

7- Al-Altwahi, H.S., Master Thesis, University of Mosul,(2014).

8- Maharaj, R., and Maharaj, C., progress in Rubber and Recycling Technology, Vol. 31. 3. (2015).

9- Dekhli, S., Mokhtar, K., A., Hammoum, F. and Bachir, D., S., Journal of Applied sciences Vol. 15, No. (3), PP. $444-455$, (2015).

10- Murshid, D.A.M, Tishreen University Journal for Research and Scientific Studies, Volume (37), No. (1), pp. 331-317, (2015).

11- Thakre, N., Mangrulkar, D., Janbandhu, M. and Saxena, J., IOSR Journal of Mechanical and Civil Engineering Vol. 13, Issue 6, PP. 120-128, (2016).

12- Gama D. A., Rosa J. M., Melo T. J. A., and Rodrigues J. K. G., Constr. Build. Mater. 106, 290 295, (2016).

13- Shirini, B., \& Imaninasab, R., Construction and Building Materials, 107, 165-171, (2016).

14- Abbas, TH.K., Saeed, S.M., Kamel, F.H., Khaled, Sh.L., and Numan, R., Iraqi Industrial Research Journal, Volume (3), No. (1), pp. 45-41, (2016).

15- Kareem, U.N.A., Diyala Journal of Engineering Sciences, Volume (9), No. (3), pp. 1-11, (2016).

16- Nekhoroshev, V. P., Nekhoroshev, S. V., Nekhorosheva, A.V., and Tarasova, O. I., Petroleum Chemistry, 57(8), 643-648. (2017).

17- Muhammad, M.K.P., Master Thesis, University of Mosul,(2017).

18- Yu, R., Zhu, X., Zhang, M., \& Fang, C. , Polymers, 10(11), 1189. (2018). 
19- Hussein, A.A., and Hamdoon, A.A., Journal of Education and Science, Volume (28), No. 4049,(2019).

20- ASTM D113-99, " Standard Test Method for Ductility of Bituminous Materials", Annual Book of ASTM Standards, Vol. 04.03, (1999).

21- ASTM D5-97, "Standard Test Method for Penetration of Bituminous Materials", Annual Book of ASTM Standards, Vol. 04.03, (2002).

22- ASTM D36-95, "Standard Test Method for Softening Point of Bitumen (Ring-and-Ball Apparatus)", Annual Book of ASTM Standards, Vol. 04.04, (2000).

23- Traxler, R. N., “ Asphalt: Its Composition, Properties in Uses”, Reinhold Publishing Co., London, p. 3. 72, (1961).

24- "Specifications of the Roads and Bridges Authority (S.O.R.B)", Republic of Iraq - Ministry of Construction and Housing - Department of Studies and Designs, Baghdad ( 2001).

25- Asphalt Institute, "Mix Design Method for Asphalt Concrete and other Hot - Mix Design". MANUAL SERIES NO. 02 (MS-2), Seventh Edition 2014.

26- ASTM D1754-97, "Standard test method for effect of heat and air on asphaltic materials (Thin Film Oven Test)", Annual Book of ASTM Standards, Vol. 04.03,(2002).

27- Brule, B., "Polymer Modified Asphalt Cement Used in the Road Construction Industry: Basic Principles", Transportation Research Board. 75th Annual Meeting. Washington, D.C., Paper No. 960167,(1996).

28- Navarro F.J., Partal P. and Martinez- Boza F ., "Thermo- Rheological behavior and storage stability of ground tire rubber-modified bitumens", Fuel,2041-2049, (2004).

29- Becker Y. ;Menderz M.p. and Rodriguez Y., Vision Technological, Vol. 9, No. 1,pp 39-50, (2001) . 
Journal of Education and Science (ISSN 1812-125X), Vol: 29, No: 3, 2020 (85-101)

30- ASTM D491-88, "Specification for Asphalt Mastic Used in Waterproofing".

31-Standard Specifications No. 1196 of 1988 issued by the Central Agency for Measurement and Quality Control of the bitumen used for flatness.

32- Salah, L.A., Master Thesis, University of Mosul. (1992). 